\title{
Prevalência do complexo teníase-cisticercose na zona rural de Matias Barbosa-MG
}

\section{Prevalence of teniasis-cysticercosis complex in a rural area of Matias Barbosa-MG}

\author{
Emilio Campos Acevedo Nieto ${ }^{1 *}$; Patrícia Santana Ferreira ${ }^{1}$; \\ Tatiane de Oliveira Santos ${ }^{1}$; Rafaella Paola Meneguete Guimarães Peixoto'; \\ Letícia Ferreira Silva ${ }^{1}$; Adriano Groppo Fellipe ${ }^{1}$; \\ Paulo Sérgio de Arruda Pinto ${ }^{2}$; Jonatas Felipe Barbosa Caldi ${ }^{3}$
}

\section{Resumo}

O complexo teníase-cisticercose é uma zoonose de grande importância para a saúde pública e animal. O ser humano desempenha importante papel sendo o único hospedeiro definitivo do parasita. Albergando no intestino as formas adultas da Taenia saginata ou da Taenia solium, cabendo à forma larvar (cisticerco) o desenvolvimento na musculatura de bovinos e suínos ou do próprio homem, quando este também atua como hospedeiro intermediário. Visando obter maior conhecimento sobre esta zoonose, o objetivo do presente estudo foi determinar o perfil epidemiológico do complexo teníase-cisticercose na zona rural do município de Matias Barbosa, localizado na Zona da Mata de Minas Gerais. Para tanto, foram realizadas coletas de sangue de 350 bovinos e 81 suínos e amostras de fezes de 124 pessoas da população presente em 74 propriedades rurais. Além disso, foi aplicado um questionário epidemiológico sobre as condições sócio-econômicas e sanitárias de cada propriedade. Observou-se uma prevalência de $0,9 \%$ de bovinos positivos ao teste de Immunoblot. Não houve suínos positivos, assim como também não foi diagnosticada pessoa portadora de teníase. Alguns fatores de risco de transmissão do complexo teníasecisticercose no município foram identificados como o consumo de carne bovina e suína mal aquecida, propriedades sem saneamento básico e criação de bovinos com acesso a fontes de contaminação. Apesar da baixa prevalência da cisticercose bovina encontrada no município de Matias Barbosa, é necessária a tomada de medidas de controle do complexo teníase-cisticercose para impedir que haja aumento de casos da doença no município, tendo em vista que foram registrados fatores favoráveis à sua manutenção.

Palavras-chave: Imunodiagnóstico, prevalência, fatores de risco, teníase-cisticercose, zoonose

\begin{abstract}
The taeniosis-cysticercosis complex is an important disease to the public and animal health. The man plays an important role in the life cycle, because he is the unique definitive host. The adult forms of the Taenia solium or Taenia saginata holds in gut, and the larvae forms develops in the muscle of bovine and swine, respectively. The objective of this study was to determine the prevalence and risk factors of the taeniosis-cysticercosis complex transmission in rural area of Matias Barbosa-MG, Brazil. Catla and swine lood and human stool samples were collected from 74 farms. Cysticercosis were eníasis were diagnosis serological methods and the teniasis by direct microscopy. Moreover, a questionnaire was
\end{abstract}

\footnotetext{
1 Discente(s), Universidade Federal de Viçosa, UFV, Viçosa, Minas Gerais, MG. E-mail: psfferreira@yahoo.com.br; ecanieto@ yahoo.com.br; tatyvetoli@hotmail.com; rafinhapaola@hotmail.com; leticiaafs@gmail.com; agroppofelippe@yahoo.com.br

Prof. da UFV, Viçosa, MG. E-mail: pintopsa@ufv.br

3 Discente, Universidade Presidente Antônio Carlos, UNIPAC, Juiz de Fora, MG. E-mail: jonatas.caldi@hotmail.com Autor para correspondência
} 
applied about the sanitary conditions of the animals and people envolved, food habits and housing. This research indicated the prevalence of $0,9 \%$ of cysticercosis bovine in the rural area of Matias Barbosa. Cases of cysticercosis swine and taeniosis were not found. Some risk factors for taeniosis-cysticercosis complex transmission in the municipality were identified and discussed. Despite the low predominance of bovine cysticercosis found in Matias Barbosa, it is necessary to maintain measures to control the taeniosis-cysticercosis complex to prevent causing increased cases of the zoonosis, considering that some risk factors favorable to their emergence were found.

Key words: Immunodiagnosis, risk factor, taeniosis-cysticercosis, prevalence, zoonosis

\section{Introdução}

A teníase-cisticercose constitui um complexo zoonótico de grande importância para a saúde pública e para o setor agropecuário. A teníase é caracterizada pela presença da forma adulta da Taenia solium ou da Taenia saginata no intestino delgado do homem, seu hospedeiro definitivo. Os bovinos ou suínos atuam como hospedeiros intermediários da $T$. saginata e da $T$. solium, respectivamente, albergando a forma larvar em seus tecidos, o que caracteriza a cisticercose. Esta também pode acometer o homem, sendo neste caso, a neurocisticercose sua forma mais grave (SILVA, 2005).

Este complexo zoonótico possui distribuição cosmopolita, causando problemas de saúde pública em várias regiões do mundo. Os agravos à saúde humana podem ser irreversíveis, quando no caso de sequelas deixadas pelos cisticercos de Taenia solium, que apresentam predileção pelo sistema nervoso (CARPIO, 2002). Quando em animais, acarretam altos custos econômicos, em função das perdas e condenação de carcaças destinadas ao consumo humano (ALMEIDA et al., 2006). Estas circunstâncias são prevalentes em países em desenvolvimento, nas regiões onde a precariedade das condições de saneamento básico e o baixo nível socioeconômico estão presentes favorecendo a ocorrência da doença (FLISSER et al., 2003).

A criação de suínos soltos ou em regime semi extensivo em locais com condições desfavoráveis de saneamento básico é também apontada como importante fator de risco para a ocorrência da cisticercose. Em um estudo realizado na China, 202 suínos foram submetidos a teste de imunodiagnóstico (ELISA) constatando uma prevalência de 2,7\% de cisticercose nestes animais (CHUNG et al., 2005). Prasad et al. (2007) também estudando a prevalência do complexo teníase-cisticercose em comunidades rurais do Norte da Índia, constataram 18,6\% (172 casos em 924). Os fatores de risco identificados foram os maus hábitos de higiene e a ingestão de carne mal passada. Hernández e Ramos (2005), realizando pesquisa semelhante em uma região de Cuba, registraram $16 \%$ de teníase (629 casos em 3.922). Paralelamente, dados colhidos em matadouros revelaram $10,6 \%$ de cisticercose bovina (448 casos em 4.207).

No Brasil, os registros existentes sobre a cisticercose bovina são basicamente procedentes de estudos realizados em matadouros. Sobre a cisticercose bovina, alguns estudos apontaram para altas taxas de prevalência em diversas regiões do país. No estado do Rio de Janeiro, uma pesquisa de campo com 60 bovinos abatidos clandestinamente constatou prevalência de $21,6 \%$ (13 casos positivos) (REZENDE et al., 2006). Em Minas Gerais, foi realizado entre 1979 a 1993 um estudo com bovinos abatidos em abatedouros de Uberlândia-MG e procedentes dos estados de MG, GO, PA, MT, TO e SP, revelando prevalência de $1,9 \%$ de cisticercose e apontando para sua presença em diferentes regiões do Brasil (REIS et al., 1996). No estado de São Paulo, foi registrado entre 1999 a 2001, 9,7\% de cisticercose entre os bovinos abatidos em diferentes abatedouros (MANHOSO; PRATA, 2004). A cisticercose suína praticamente não é reportada em 
vista do manejo tecnificado adotado nas granjas, que controla efetivamente a doença. Contudo, sabese que ainda coexistem no país grande consumo de carnes, de bovinos e suínos, não inspecionadas, principalmente no interior dos estados. Em muitos municípios predominam o consumo de carne de suíno criados soltos e de bovinos provenientes de abate clandestino (observação pessoal). Pinto et al. (2002) ressaltam que a prevalência em animais de criações domésticas pode ser 5 vezes maior que a dos animais criados em sistemas tecnificados.

Os dados sobre a prevalência da cisticercose animal em Minas Gerais são na maioria obtidos pelos serviços oficiais de Inspeção Veterinária nos matadouros (ALMEIDA et al., 2002, 2006). Raras são as informações baseadas em dados de campo oriundos de animais vivos. Dessa forma, a situação atual da teníase e da cisticercose é pouco conhecida em diversas regiões do estado e país. Estes fatos somados a não obrigatoriedade de notificação e à ausência de programas de controle na maioria dos estados, tornam de grande importância o desenvolvimento de estudos epidemiológicos destas zoonoses, a fim de conhecer a sua realidade sanitária e direcionar as ações de controle.

Nesta perspectiva, o objetivo do presente estudo foi avaliar o perfil epidemiológico do complexo teníase-cisticercose na zona rural do município de Matias Barbosa, Minas Gerais, de acordo com as condições locais de moradia dos humanos e de criação dos suínos e bovinos.

\section{Material e Métodos}

Foi realizado um estudo epidemiológico transversal no período de janeiro a agosto de 2010, em propriedades rurais do município de Matias Barbosa, localizado na Zona da Mata do Estado de Minas Gerais. O município possui população de 13.435 habitantes e de 8.589 bovinos, criados em 123 propriedades rurais (IMA, 2009a, 2009b; IBGE, 2010).
As propriedades estudadas foram sorteadas aleatoriamente, considerando os seguintes parâmetros: prevalência estimada de $2 \%$ de propriedades positivas para a cisticercose (baseada em resultado registrado em comunidades rurais do município de Tumiritinga-MG) (ACEVEDO NIETO, 2011), precisão de $2 \%$ e intervalo de confiança de 95\%. O cálculo do tamanho da amostra foi realizado utilizando o programa EpiInfo versão 3.5.1 (CDC, 2008), resultando no total de 74 propriedades que foram amostradas.

Devido à indisponibilidade de informação sobre o número de suínos de criação de fundo de quintal presentes na zona rural do município, foram consideradas no cálculo da quantidade de unidade amostral as propriedades contendo bovinos. Como critério de seleção, apenas os animais com idade igual ou superior a três meses foram amostrados, a fim de evitar a ocorrência de falsos positivos visto que animais muito jovens podem apresentar anticorpos transferidos pela mãe. $\mathrm{Na}$ propriedade onde não havia criação de suínos, recorreu-se à propriedade vizinha. Dessa forma, obtiveram-se amostras de sangue de 350 bovinos e 81 suínos.

As amostras dos animais foram obtidas porpunção da veia caudal dos bovinos e do plexo orbitário dos suínos. Estas amostras foram dessoradas à temperatura ambiente e logo em seguida estocadas a $-20^{\circ} \mathrm{C}$. O diagnóstico sorológico da cisticercose animal foi realizado por triagem pelo teste ELISA indireto, sendo os casos positivos submetidos ao Immunoblot para confirmação e resultado final (PINTO et al., 2000, 2001; MONTEIRO; PINTO; DIAS, 2006). Para o teste de ELISA indireto as placas de poliestireno foram sensibilizadas com antígeno heterólogo, de cisticercos de Taenia crassiceps, que apresenta reação com os anticorpos para cisticercos de Taenia solium e Taenia saginata. Para a sensibilização, o antígeno foi diluído em solução tamponada de carbonato-bicarbonato 0,5M pH 9,6 na concentração de 1:12,5 (suínos) e $1: 25$ (bovinos) e incubadas por 1 hora a $37^{\circ} \mathrm{C}$. Após lavagens em solução salina contendo $0,05 \%$ 
de Tween-20 foi realizado o bloqueio dos sítios reativos com solução contendo leite desnatado a $5 \%$ em PBS pH 7,4, durante 1 hora a $37^{\circ} \mathrm{C}$. Novas lavagens foram realizadas e as amostras de soro diluídas em leite desnatado a 1\% em PBS pH 7,4 e a placa incubada por 30 minutos a $37^{\circ} \mathrm{C}$. Após lavagens, foram adicionados os conjugados antiIgG de bovino A-5295 e anti-IgG de suíno A-5670 nas concentrações de 1:5000 (Sigma Chemical Co, St. Louis, MO, USA) diluídos e repetidos os procedimentos de incubação e lavagem. A reação foi em seguida revelada com solução de OPD $(0,1 \%)$ e $\mathrm{H}_{2} \mathrm{O}_{2} 0,03 \%$ em tampão citrato-fosfato $0,2 \mathrm{M} \mathrm{pH} 5,0$, durante incubação por 5 minutos a $37^{\circ} \mathrm{C}$. A reação foi então bloqueada com $\mathrm{H}_{2} \mathrm{SO}_{4} 4 \mathrm{~N}$ e as leituras realizadas em espectrofotômetro a $492 \mathrm{~nm}$. Em cada etapa a quantidade de reagentes aplicados à placa foi de $100 \mu$, exceto para a solução bloqueadora, $200 \mu 1$. A fim de minimizar as diferenças entre as placas, facilitar a comparação e fornecer um resultado mais exato, o ponto de corte (Cut-off) foi calculado a partir das médias dos valores das densidades ópticas (DO) dos controles negativos de cada placa, acrescido de dois desvios-padrão (2DP). As DO obtidas foram ajustadas pelo fator de correção, que foi calculado a partir da diferença entre as médias dos controles negativos e positivos da placa padrão dividido pela diferença entre as médias dos controles negativos e positivos da placa teste.

Para o teste de Immunoblot, os peptídeos separados por SDS-PAGE foram transferidos do gel para membranas de nitrocelulose de $0,2 \mu \mathrm{c}$ (Millipore, USA), segundo metodologia descrita por Towbin et al. (1979), utilizando solução tamponada de transferência contendo metanol (Trishidroximetilaminoetano $25 \mathrm{mM}$, glicina $192 \mathrm{mM}$ e metanol $20 \% \mathrm{v} / \mathrm{v}, \mathrm{pH} 8,3$ ). A transferência foi procedida por um período de 12 horas à temperatura de $4^{\circ} \mathrm{C}$, com corrente de $150 \mathrm{~mA}$ e voltagem constante de 50V (Bio-Rad Laboratories, Hercules, CA, USA). Após a transferência, as membranas de nitrocelulose foram coradas em solução aquosa contendo Ponceau-S a $0,5 \%$, para visualização qualitativa e quantitativa da transferência. A partir das membranas foram obtidas tiras de 3 a $4 \mathrm{~mm}$ de largura que foram descoradas por três lavagens consecutivas em solução salina $(\mathrm{NaCl} 0,15 \mathrm{M})$ contendo $0,05 \% \mathrm{v} / \mathrm{v}$ de Tween 20 . As tiras foram tratadas com solução bloqueadora contendo leite desnatado (Molico-Nestlé) a 5\% dissolvido em Trissalina (Tris-hidroximetilaminoetano $10 \mathrm{mM}$ e $\mathrm{NaCl}$ $0,15 \mathrm{M}, \mathrm{pH} 7,4)$ por aquecimento até cerca de $90^{\circ} \mathrm{C}$ e filtrado em papel de filtro à temperatura ambiente. As amostras de soros nas concentrações de 1:100 (suínos) e 1:25 (bovinos), foram acrescentadas à solução diluidora (Solução bloqueadora diluída a $1 / 5$ em Tris-salina), adicionadas às tiras e incubadas à temperatura ambiente sob agitação lenta em mesa oscilante durante 1 hora. Após seis lavagens de cinco minutos cada, as tiras foram novamente incubadas por uma hora com os conjugados antiIgG de bovino A-5295 (concentração de 1:2000) e anti-IgG de suíno A-5670 (concentração de 1:1000) (Sigma Chemical Co, St. Louis, MO, USA), diluídos e após o período novas lavagens foram procedidas. Os peptídeos reativos foram evidenciados com a solução cromógena (Diaminobenzidina 5mg, H2O2 1,5\% em PBS pH 7,2) por cerca de 10 minutos, em seguida as tiras foram lavadas com água destilada e secadas. Os referidos peptídeos foram analisados com o auxílio do programa Quantity One, versão 1.4 (Bio-Rad). Os marcadores de peso molecular utilizados evidenciaram as bandas que variaram de $205 \mathrm{kD}$ a $6,5 \mathrm{kD}$ (Sigma M-4038). Em ambos os testes foi utilizado o antígeno total de Taenia crassiceps, na concentração de $6 \mu \mathrm{g} / \mathrm{mm}$ conforme recomendações de Pinto et al. (2001).

Para a pesquisa dos portadores de teníase, foram coletadas 124 amostras de fezes da população humana residente nas mesmas propriedades, que foram submetidas ao exame parasitológico pela pesquisa de ovos do parasita utilizando o método de Hoffman (SILVA, 2005). Além disso, foi aplicado em todas as propriedades um questionário, visando obter informações sobre as condições sócioeconômicas e sanitárias de cada propriedade. 
A coleta de sangue dos animais, fezes dos moradores e aplicação do questionário foram realizadas após a concordância dos participantes, com a assinatura do Termo de Consentimento Livre e Esclarecido. O presente estudo foi aprovado pelo Comitê de Ética em Pesquisa com Seres Humanos - Processo 34/2009 e Comissão de Ética para Uso de Animais - Processo 38/2010 vinculados à Universidade Federal de Viçosa. Os dados obtidos através da aplicação dos questionários e os resultados laboratoriais foram sistematizados no programa Epi Info, versão 3.5.1 (CDC, 2008), e com os mesmos realizada análise descritiva das variáveis e o cálculo da prevalência de cisticercose bovina e suína e de teníase.

\section{Resultados e Discussão}

A prevalência da cisticercose bovina foi de 0,9\% (3/350), em três propriedades distintas. Este resultado assemelhou-se ao encontrado na zona rural de Viçosa-MG também localizado na Zona da Mata Mineira, onde foi desenvolvida uma pesquisa com a mesma metodologia deste estudo, observando uma prevalência de $0,42 \%$ de cisticercose bovina (SANTOS, 2010). Ao contrário destes resultados, Acevedo Nieto (2011) utilizando metodologia semelhante, verificou na zona rural do município de Tumiritinga, região leste do estado de Minas Gerais, uma prevalência de $8,8 \%$ em bovinos e $5,2 \%$ em suínos.

Devido ao pequeno número de bovinos positivos para cisticercose e da ausência de casos de cisticercose suína e de teníase registrados, não foi possível realizar análise de associação entre a e as características sócio-econômicas coletadas, e consequentemente, análise de possíveis fatores de risco (Odds Ratio) para a transmissão desta zoonose. Situação analítica semelhante foi deparada por Phiri et al. (2002) e Santos (2010) ao estudarem a prevalência do complexo teníase cisticercose em outras localidades.
Algumas características sabidamente conhecidas como desfavoráveis à manutenção desta parasitose, encontradas nas propriedades amostradas podem ter contribuído para a baixa prevalência da cisticercose bovina no município de Matias Barbosa-MG. Com relação à origem da água consumida pelos animais, observou-se que na maioria das propriedades $74,4 \%$ a água foi proveniente de mina/nascente, $9 \%$ de cisterna, $2,6 \%$ de poço artesiano, $6,4 \%$ água tratada pelo Órgão responsável no município e em apenas $7,7 \%$ a água provinha de ribeirão. Boone et al. (2007) analisando fatores de risco para a cisticercose bovina na Bélgica, verificaram que bovinos com livre acesso a rios e lagos apresentaram quase duas vezes mais chance de apresentarem cisticercose; favorecendo a hipótese de que a água contaminada com ovos de Taenia sp. possa atuar como fator de risco para a infecção dos animais. No presente estudo, em $7,7 \%$ das propriedades os bovinos bebiam água de ribeirão, o que possivelmente influenciou na baixa frequência de cisticercose observada. Apesar do baixo número de bovinos positivos para cisticercose em Matias Barbosa, é importante ressaltar que a doença está presente no município.

Foi possível verificar nas propriedades estudadas características que permitem a disseminação de ovos do parasita, o que possivelmente tem contribuído para a presença de animais positivos. A falta de saneamento básico encontrada na maioria das localidades $(60,3 \%)$ pesquisadas, representa riscos para o aparecimento do complexo teníasecisticercose, pois permite o acesso dos animais aos dejetos humanos contendo ovos do parasito e conseqüentemente o desenvolvimento da cisticercose, podendo significar maiores riscos que em situações onde há destinação correta de dejetos humanos, como em fossas, por exemplo. Todos os bovinos pesquisados permaneciam grande parte do tempo soltos, o que permitia o contato destes animais com pastagens favorecendo a infecção, desta forma, poderia-se especular que as áreas estudadas apresentavam uma baixa contaminação por ovos de $T$. saginata. Com relação ao destino 
do esgoto verificou-se que ainda apenas 39\% das propriedades fazem destinação em fossas isoladas, tendo as demais o destino a céu aberto ou em pequenos cursos de água.

Não foram encontrados suínos positivos para a cisticercose. Esse fato pode ter ocorrido devido à presença de algumas características encontradas nas propriedades estudadas, que são consideradas como favoráveis ao seu controle. Uma dessas condições é o sistema de criação dos suínos, onde em 77,3\% das propriedades os animais permaneciam sempre presos em chiqueiros ou cercados e em apenas $22,7 \%$ utilizava-se o sistema de criação misto, onde os animais mais jovens permaneciam soltos na propriedade e apenas os adultos presos. Widdowson et al. (2000) verificaram que as famílias que criavam suínos soltos apresentavam 2,3 vezes mais chance de possuir animais positivos para a cisticercose e aquelas que defecavam a céu aberto apresentaram chance de 1,4. Pouedet et al. (2002), observaram diferença significativa entre a prevalência de cisticercose em suínos criados soltos e dos criados presos. Segundo Gottschalk (2006), a criação de suínos soltos e o destino inadequado das fezes humanas são importantes fatores de risco para a cisticercose suína.

Todas as amostras de fezes humanas resultaram negativas para teníase durante o diagnóstico coproparasitológico. Resultado semelhante foi encontrado por Iasbik (2010) no município de Viçosa-MG. A ausência de indivíduos com teníase na zona rural do município de Matias Barbosa pode estar associada à presença de fatores considerados favoráveis ao controle desta zoonose. Por outro lado, este resultado pode também estar relacionado ao método de diagnóstico empregado, visto que as amostras foram coletas uma única vez pelos participantes da pesquisa, não descartando a possibilidade da presença do parasito no organismo humano.
Algumas características observadas na população podem ter favorecido os achados. Foi verificado que as famílias analisadas possuíam o hábito de realizar tratamento regular com antiparasitários. Por ser um medicamento de baixo custo, os antiparasitários são facilmente adquiridos pela população, contribuindo para a diminuição ou até mesmo ausência de algumas parasitoses intestinais. Outra interessante informação obtida foi que o Programa de Saúde da Família (PSF) atua no município junto às comunidades rurais, com cobertura de atendimento de $100 \%$ da população.

Ao analisar o consumo de carne, característica fundamental na transmissão da teníase, verificouse que o consumo de carne bovina foi comum em $97,4 \%$ das propriedades, sendo que $63,2 \%$ das famílias destas propriedades possuíam o hábito de consumi-la mal passada. Com relação à origem da carne bovina consumida em $75 \%$ das propriedades foi proveniente de açougues e mercados, em 6,6\% da própria propriedade e em $8,4 \%$ de ambas as fontes. Com relação ao consumo de carne suína, $85,9 \%$ das famílias disseram consumi-la, e destas, $4,5 \%$ afirmaram consumir carne suína mal passada. Quanto à origem da carne suína 25,4\% revelaram que obtinham a carne dos animais criados e abatidos na propriedade, $68,7 \%$ consumiam a carne proveniente de açougues e mercados e $6 \%$ consumiam a carne proveniente de ambos os locais. Sabe-se que o consumo de carne suína ou bovina mal passada é um importante fator responsável pela transmissão da teníase, caso haja cisticercos viáveis na carne consumida. Wandra et al. (2006) verificaram que todos os indivíduos positivos para a teníase revelaram consumir carne suína ou bovina crua, reforçando a importância deste hábito na transmissão desta parasitose. Apesar do pequeno número de bovinos positivos na zona rural de Matias Barbosa é importante ressaltar que a doença está presente no município necessitando de atenção pelos programas de saúde publica. 


\section{Conclusões}

A população da zona rural do município de Matias Barbosa-MG apresentou baixa prevalência de cisticercose bovina $(0,9 \%)$, e ausência de casos de cisticercose suína e de teníase. As propriedades apresentaram fatores favoráveis ao controle do complexo teníase-cisticercose, como a criação de suínos presos, hábito dos indivíduos consumirem antiparasitários regularmente, destino adequado do lixo, água de boa qualidade destinada ao consumo animal. Ao mesmo tempo, verificaramse características consideradas propícias para o desenvolvimento desta zoonose como consumo de carne bovina e suína mal aquecida, propriedades sem saneamento básico e criação de bovinos com acesso a fontes de contaminação. Estes achados reforçam necessidade de medidas de controle no município. Portanto, torna-se importante uma articulação estratégica entre os serviços de vigilância epidemiológica, secretaria da agricultura e secretaria de obras e de educação e comunidades rurais, na finalidade de sensibilizar a população rural sobre os problemas decorrentes desta zoonose e atuar de forma a impedir a difusão, estabelecimento e manutenção do ciclo nas comunidades rurais.

\section{Agradecimentos}

Os autores agradecem à Fundação de Apoio à Pesquisa do Estado de Minas Gerais (FAPEMIG) e ao Conselho Nacional de Desenvolvimento Científico e Tecnológico - CNPq pelo financiamento da respectiva pesquisa e ao Instituto Mineiro de Agropecuária, pelo suporte técnico no planejamento e na execução dos trabalhos de campo.

\section{Referências}

ACEVEDO NIETO, E. C. Perfil epidemiológico do complexo teníase-cisticercose na zona rural do município de Tumiritinga-MG. 2011. Dissertação (Mestrado em Medicina Veterinária) - Universidade Federal de Viçosa, Viçosa.
ALMEIDA, L. P.; MOREIRA, M. D.; REIS, D. O.; SANTOS, W. L. M. Cisticercose bovina: um estudo comparativo entre animais abatidos em frigoríficos com serviço de inspeção federal e com inspeção municipal. Revista Higiene Alimentar, São Paulo, v. 16, n. 99, p. 5155, 2002.

ALMEIDA, L. P.; REIS, D. O.; MOREIRA, M. D.; PALMEIRA, S. B. S.Cisticercose em bovinos procedentes de Minas Gerais e abatidos em frigoríficos de Uberlândia-MG, no período de 1997 a 2001. Revista Higiene Alimentar, São Paulo, v. 2, n. 139, p. 40-43, 2006.

BOONE, I.; THYS, E.; MARCOTTY, T.; BORCHGRAVE, J.; DUCHEYNE, E.; DORNY, P. Distribution and risk factors of bovine cysticercosis in Belgian dairy and mixed herds. Preventive Veterinary Medicine, Fort Collins, USA, n. 82, p. 1-11, 2007.

CARPIO, A. Neurocysticercosis: an update. The Lancet Infectious Disease, London, v. 2, n. 12, p. 751-62, 2002.

CENTER FOR DISEASE CONTROL AND PREVENTION - CDC. EpiInfo. Database and statistics software for public health professionals. Versão 3.5.1. Atlanta: USA, 2008. Disponível em: <http://wwwn.cdc. gov/epiinfo/>. Acesso em: 20 jan. 2011.

CHUNG, J. Y.; EOM, K. S.; YANG, Y.; LI, X.; FENG, Z.; RIM, H. J.; CHO, S. Y.; KONG, Y. A. seroepidemiological survey of Taenia soliumcysticercosis in Nabo, Guangxi Zhuang Autonomous Region, China. Korean Journal of Parasitology, Seoul, v. 43, n. 4, p. 135-139, 2005.

FLISSER, A.; SARTI, E.; LIGHOWLERS, M.; SCHANTZ, P. Neurocysticercosis: regional status, epidemiology, impact and control measures in the Americas. Acta Tropica, Canada, n. 87, p. 43-51, 2003.

GOTTSCHALK, S.; BUZI, K. A.; GALINDO, L. A.; ABREU, B. X.; NUNES, C. M.; BIONDI, G. F. Soroprevalência e aspectos epidemiológicos da cisticercose suína em criações de "fundo de quintal" na microrregião de Registro - SP. Veterinária e Zootecnia, São Paulo, v. 13, n. 2, p. 192-200, 2006.

HERNÁNDEZ, M. S.; RAMOS, M. S. Epidemiología del complexo Taenia saginata y Cisticercus bovis en La Província Ciego de Ávila, Cuba. Revista de Patologia Tropical, Goiânia, v. 34, n. 1, p. 43-52, 2005.

IASBIK, A. F.; PINTO, P. S. A.; BEVILACQUA, P. D.; NERO, L. A.; SANTOS, T. O.; FELIPPE, A. G. Prevalência do complexo teníase-cisticercose na zona rural do município de Viçosa, Minas Gerais. Ciência Rural, Santa Maria, v. 40, n. 7, p. 1664-1667, 2010. 
INSTITUTO BRASILEIRO DE GEOGRAFIA E ESTATÍSTICA - IBGE. Censo 2010. 2010. Disponível em: <http://www.ibge.gov.br>. Acesso em: 20 jan. 2011.

INSTITUTO MINEIRO DE AGROPECUÁRIA IMA. Secretaria Estadual de Agricultura, Pecuária e Abastecimento de Minas Gerais. Sistema de Defesa Agropecuária (SIDAGRO, 2009). 2009a. Disponível em: <www.sidagro.ima.mg.gov.br>. Acesso em: 02 jan. 2009.

Secretaria Estadual de Agricultura, Pecuária e Abastecimento de Minas Gerais. Gerência de inspeção de produtos de origem animal. Mapas nosográficos. Minas Gerais: IMA, 2009. 2009b.

MANHOSO, F .F. R.; PRATA, L. F. Prevalência da cisticercose bovina na região oeste do estado de São Paulo. Revista Higiene Alimentar, São Paulo, v. 18, n. 121, p. 42-49, 2004.

MONTEIRO, L. L; PINTO, P. S. A.; DIAS, F. S. Evaluation of the ELISA test for the antibody detection in cattle naturally and experimentally infected with Cysticerccus bovis. Veterinary Parasitology, Amsterdam, n. 141, p. $260-263,2006$.

PHIRI, I. K.; DORNY, P.; GABRIEL, S.; WILLINGHAM, A. L.; SPEYBROECK, N.; VERCRUYSSE, J. The prevalence of porcine cysticercosis in Eastern and Southern provinces of Zambia. Veterinary Parasitology, Amsterdam, n. 108, p. 31-39, 2002.

PINTO, P. S. A.; VAZ, A. J.; NAKAMURA, P. M.; GERMANO, P. M. L. Immunoblot analisys using antigens from Taenia crassiceps cysticerci in the diagnosis of swine cysticercosis. Boletin Chileno de Parasitologia, Santiago, v. 56, n. 1-2, p. 36-42, 2001.

PINTO, P. S. A.; VAZ, A. J.; GERMANO, P. M. L.; NAKAMURA, P. M. Performance of the ELISA test for swine cysticercosis using antigens of Taenia solium and Taenia crassiceps cysticerci. Veterinary Parasitology, Amsterdam, n. 88, p. 127-130, 2000.

POUEDET, M. S. R.; ZOLI, A. P.; NGUEKAMA, VONDOU, L.; ASSANA, E.; SPEYBROECK, N.; BERKVENS, D.; DORNY, P.; BRANDT, J.; GEERTS, S. Epidemiological survey of swine cysticercosis in two rural communities of West-Cameroon. Veterinary Parasitology, Amsterdam, n. 106, p. 45-54, 2002.
PRASAD, K. N.; PRASAD, A.; GUPTAB, R. K.; PANDEYC, C. M.; SINGHC, U. Prevalence and associated risk factors of Taenia solium taeniasis in a rural pig farming community of north India. Transactions of the Royal Society of Tropical Medicine and Hygiene, London, n. 101, p. 1241-1247, 2007.

REIS, D. O.; MUNDIM, M. J. S.; CABRAL, D. D. Cisticercose bovina 15 anos de ocorrência em animais abatidos em Uberlândia, MG, Brasil. Revista Higiene Alimentar, São Paulo, v. 10, n. 43, p. 373-9, 1996.

REZENDE, R. B. C.; FERNANDEZ, A. T.; COSTA, F.; SILVA, T. J. P. Ocorrência de cisticercose em bovinos abatidos clandestinamente no município de Silva Jardim, RJ. Revista Higiene Alimentar, São Paulo, v. 21, n. 140, p. 103-9, 2006.

SANTOS, T. O. Levantamento epidemiológico do complexo teníase-cisticercose na zona rural do município de Viçosa-MG. 2010. Dissertação (Mestrado em Medicina Veterinária) - Universidade Federal de Viçosa, Viçosa.

SILVA, A. V. M. Teníase e cisticercose, In: NEVES, D. P. et al. 11. ed. Parasitologia humana. São Paulo: Atheneu, 2005. cap. 25, p. 227-237.

TOWBIN, H.; STAEHELIN, T.; GORDON, I. Eletrofhoretic transfer of proteinsfron polyacrylamide gels to nitrocellulose sheets: procedure and some applications. Proceedings of the National Academy of Sciences of the United States of America, Washington, v. 76, n. 9, p. 4350-4, 1979.

WANDRA, T.; SUTISNAC, P.; DHARMAWAND, N. S.; MARGONOE, S. S.; SUDEWI, R.; SUROSOA, T.; CRAIGG, P. S.; ITO, A. High prevalence of Taenia saginata taeniasis and status of Taenia solium cysticercosis in Bali, Indonesia, 2002-2004. Transactions of the Royal Society of Tropical Medicine and Hygiene, London, n. 100, p. 346-353, 2006.

WIDDOWSON, M. A.; COOKZ, A. J. C.; WILLIAMS, J. J.; RODRIGUEZ, R.; ARGAES, F.; RODRIGUEZ, I.; DOMINGUEZ, J. L. Investigation of risk factors for porcine Taenia solium ysticercosis: a multiple regression analysis of a cross-sectional study in the Yucatan Peninsula, Mexico. Transactions of the Royal Society of Tropical Medicine and Hygiene, London, n. 94, p. 620$624,2000$. 